\title{
Pattern Formation in Predator-Prey Model with Delay and Cross Diffusion
}

\author{
Xinze Lian, ${ }^{1,2}$ Shuling Yan, ${ }^{3}$ and Hailing Wang ${ }^{4}$ \\ ${ }^{1}$ Department of Mathematics, Wenzhou University, Wenzhou 325035, China \\ ${ }^{2}$ Chengdu Institute of Computer Application, Chinese Academy of Sciences, Chengdu 610041, China \\ ${ }^{3}$ College of Mathematics and Econometrics, Hunan University, Changsha 410082, China \\ ${ }^{4}$ Department of Mathematics, Hubei Minzu University, Enshi 445000, China
}

Correspondence should be addressed to Xinze Lian; xinzelian@163.com

Received 29 August 2013; Revised 4 October 2013; Accepted 4 October 2013

Academic Editor: Massimiliano Ferrara

Copyright (C) 2013 Xinze Lian et al. This is an open access article distributed under the Creative Commons Attribution License, which permits unrestricted use, distribution, and reproduction in any medium, provided the original work is properly cited.

\begin{abstract}
We consider the effect of time delay and cross diffusion on the dynamics of a modified Leslie-Gower predator-prey model incorporating a prey refuge. Based on the stability analysis, we demonstrate that delayed feedback may generate Hopf and Turing instability under some conditions, resulting in spatial patterns. One of the most interesting findings is that the model exhibits complex pattern replication: the model dynamics exhibits a delay and diffusion controlled formation growth not only to spots, stripes, and holes, but also to spiral pattern self-replication. The results indicate that time delay and cross diffusion play important roles in pattern formation.
\end{abstract}

\section{Introduction}

The spatial component of ecological interaction has been identified as an important factor in how ecological communities are shaped. Understanding the role of space is challenging both theoretics and empirism [1]. Empirical evidence suggests that the spatial scale and structure of environment can influence population interactions [2]. And pattern formation in nonlinear complex systems is one of the central problems of the natural, social, and technological sciences [3-12]. The classical approach to the origin of spatial structures was developed by Turing [13]. Understanding of patterns and mechanisms of species spatial dispersal is an issue of significant current interest in conservation biology and ecology. It arises from many ecological applications; particularly, it plays a major role in connection to biological invasion and disease spreading $[14,15]$.

In recent years, one of the important predator-prey modelsis Leslie-Gower model. A modified version of reaction- diffusion Leslie-Gower predator-prey model with Hollingtype II functional response takes the form $[16,17]$

$$
\begin{gathered}
\frac{\partial u}{\partial t}=u(r-a u)-\frac{c_{1} u v}{b_{1}+u}+d_{1} \nabla^{2} u, \\
\frac{\partial v}{\partial t}=v\left(d-\frac{c_{2} v}{b_{2}+u}\right)+d_{2} \nabla^{2} v,
\end{gathered}
$$

where $u$ and $v$ are prey and predator densities, respectively. $r, a, d, b_{i}$, and $c_{i}(i=1,2)$ are positive constants. $r$ is the growth rate of preys, $d$ is the growth rate of predators, $a$ is the strength of competition among individuals of species $u, b_{1}$ (resp., $b_{2}$ ) measures the extent to which environment provides protection to prey $u$ (resp., to the predator $v$ ), and $c_{1}$ is the maximum value of the per capita reduction of $u$ due to $v$. $c_{2}$ has a similar meaning to $c_{1} \cdot d_{1}$ and $d_{2}$ are the diffusion coefficients that imply the movement of individuals from a higher to a lower concentration region. $\nabla^{2}=\left(\partial^{2} / \partial x^{2}\right)+$ $\left(\partial^{2} / \partial y^{2}\right)$ is the usual Laplacian operator in two-dimensional space. 
For model (1), Camara and Aziz-Alaoui [18-20] paid more attention to pattern formation in the spatial domain and observed the labyrinth, chaos, and spiral wave patterns. In addition, Guan et al. [21] studied model (1) incorporating a refuge protecting $m u$ of the prey in the case of $c_{1}=c_{2} \neq 0$ and $b_{1}=b_{2} \neq 0$, where $m \in[0,1)$ is a constant. This leaves $(1-m) u$ of the prey available to the predator, and model (1) becomes

$$
\begin{aligned}
\frac{\partial u}{\partial t} & =u(r-a u)-\frac{(1-m) u v}{b+(1-m) u}+d_{11} \nabla^{2} u \\
& \triangleq f(u, v)+d_{11} \nabla^{2} u \\
\frac{\partial v}{\partial t} & =v\left(d-\frac{c v}{b+(1-m) u}\right)+d_{22} \nabla^{2} v \\
& \triangleq g(u, v)+d_{22} \nabla^{2} v \\
& \triangleq v(d-h(u, v))+d_{22} \nabla^{2} v .
\end{aligned}
$$

In [21], the authors proved the global stability of the positive equilibria, determined the Turing space in the spatial domain, and performed a series of numerical simulations and found that model (2) exhibits complex Turing pattern replication: spots, stripes, and holes patterns.

On the other hand, a time delay occurs naturally in just about every interaction of the real world. The original motivations for studying delay differential equations mainly come from their applications in feedback control theory [2229]. And time delay also is an ubiquitous phenomenon in biological systems and has an important role in affecting population dynamics. In a system described by ordinary differential equations, time delay could change qualitatively the nature of the equilibrium from a stable equilibrium to an unstable one and thus induces bifurcation [30]. For a predator-prey system, time delay was often realized as feedback. The effect of this kind of delay on the dynamical behavior of populations has been studied by a number of papers [31-33]. Because of the importance of time delay to control dynamics of a system, a number of works have been done on effect of delay on the spatial-temporal system [34$44]$. In our previous work [43], we studied the spatiotemporal complexity of a Leslie-Gower model incorporating a prey refuge with time delay effect:

$$
\begin{aligned}
\frac{\partial u}{\partial t} & =u(r-a u)-\frac{(1-m) u v}{b+(1-m) u}+d_{11} \nabla^{2} u \\
& \triangleq f(u, v)+d_{11} \nabla^{2} u, \\
\frac{\partial v}{\partial t} & =v\left(d-\frac{c v(t-\tau)}{b+(1-m) u(t-\tau)}\right)+d_{22} \nabla^{2} v \\
& \triangleq v\{d-h[u(t-\tau), v(t-\tau)]\}+d_{22} \nabla^{2} v,
\end{aligned}
$$

where $\tau>0$ is a constant delay due to gestation and $u(x, y, t-\tau)$ and $v(x, y, t-\tau)$ are densities of prey and predator, respectively, at moment $t-\tau$ and position $(x, y)$. For simplicity, set $u(x, y, t-\tau):=u(t-\tau), v(x, y, t-\tau):=v(t-\tau)$. In [43], we analyzed the effects of delay and diffusion on the spatiotemporal dynamics of model (3) and gave the pattern formation via numerical simulation.

Furthermore, in nature, there is a tendency that the preys would keep away from predators and the escape velocity of the preys may be taken as proportional to the dispersive velocity of the predators [45-49]. In the same manner, there is a tendency that the predators would get closer to the preys and the chase velocity of predators may be considered to be proportional to the dispersive velocity of the preys. Keeping these in view, cross-diffusion arises, which was proposed first by Kerner [50] and first applied in competitive population system by Shigesada et al. [51]. And there comes a question: how does cross-diffusion affect pattern formation in a delayed Leslie-Gower predator-prey model incorporating a prey refuge?

In this paper, we mainly focus on the effect of crossdiffusion on the pattern formation in the following LeslieGower predator-prey model incorporating a prey refuge:

$$
\begin{aligned}
\frac{\partial u}{\partial t}= & f(u, v)+d_{11} \nabla^{2} u+d_{12} \nabla^{2} v \\
\frac{\partial v}{\partial t}= & v\{d-h[u(t-\tau), v(t-\tau)]\} \\
& +d_{21} \nabla^{2} u+d_{22} \nabla^{2} v
\end{aligned}
$$

where the cross-diffusion coefficients $d_{12}$ and $d_{21}$ express the respective population fluxes of the preys and predators resulting from the presence of the other species, respectively. Other parameters are the same definitions as model (3). And we call

$$
D=\left(\begin{array}{ll}
d_{11} & d_{12} \\
d_{21} & d_{22}
\end{array}\right)
$$

the diffusive matrix and assume that $d_{11}>0, d_{22}>0$, and $\operatorname{det}(D)=d_{11} d_{22}-d_{12} d_{21}>0$.

Following [44], we explain the meaning and units of each variable and constant in model (4).

(i) The prey $u$ and predator $v$ are usually measured in $\mathrm{mg}$ of dry weight per liter [mg.dw/l].

(ii) Time $t$ and time delay $\tau$ are measured in days $[d]$.

(iii) The length of $x, y \in(0, L)$ is measured in meter [m].

(iv) $r$ is the growth rate of prey $u$ and $d$ is the growth rate of predator $v$, measured in $\left[\mathrm{d}^{-1}\right]$.

(v) $c$ is the maximum value of the per capita reduction of $v$ due to $u$, which is not available in abundance and is measured in $\left[\mathrm{d}^{-1}\right]$.

(vi) $a$ describes the strength of competition among individuals of species $u$ and is measured in [( $\mathrm{mg}$. $\left.\mathrm{dw} / \mathrm{l})^{-1} \mathrm{~d}^{-1}\right]$.

(vii) $b$ describes the extent to which environment provides protection to prey $u$ and predator $v$ and is usually measured in mg of dry weight per liter [mg.dw/l].

(viii) $d_{i j}>0(i, j=1,2)$ are the diffusion coefficients corresponding to the prey and predator, respectively, and are measured in $\left[\mathrm{m}^{2} \mathrm{~d}^{-1}\right]$. 
Model (4) is to be analyzed under the following nonzero initial conditions:

$$
\begin{aligned}
u\left(x, y, t_{0}\right) & >0, \quad v\left(x, y, t_{0}\right)>0, \\
(x, y) & \in \Omega=(0, L) \times(0, L), \\
t_{0} & \in[-\tau, 0],
\end{aligned}
$$

and zero-flux boundary conditions:

$$
\left.\frac{\partial u}{\partial n}\right|_{\partial \Omega}=\left.\frac{\partial v}{\partial n}\right|_{\partial \Omega}=0
$$

In the above, $L$ denotes the size of the system in square domain, and $n$ is the outward unit normal vector of the boundary $\partial \Omega$. The main reason for choosing such boundary conditions is that we are interested in the self-organization of pattern, and zero-flux conditions imply no external input [5].

This paper is organized as follows. In the next section, we mainly present the Hopf and Turing bifurcation analysis. In Section 3, we perform a series of numerical simulation to show evolution process of prey $u$. And in the last section, we give some concluding remarks.

\section{Bifurcation Analysis}

In this section, we will focus on the conditions of Hopf and Turing bifurcation. Following $[40,43]$, if time delay $\tau$ is small, one can expand $u(x, y, t-\tau)$ and $v(x, y, t-\tau)$ as

$$
\begin{aligned}
& u(x, y, t-\tau)=u(x, y, t)-\tau \frac{\partial u(x, y, t)}{\partial t} \\
& v(x, y, t-\tau)=v(x, y, t)-\tau \frac{\partial v(x, y, t)}{\partial t}
\end{aligned}
$$

Substituting (8) into (4), we obtain

$$
\begin{gathered}
\frac{\partial u}{\partial t}=f(u, v)+d_{11} \nabla^{2} u+d_{12} \nabla^{2} v \\
\frac{\partial v}{\partial t}=v\left[d-h\left(u(x, y, t)-\tau \frac{\partial u(x, y, t)}{\partial t},\right.\right. \\
\left.\left.v(x, y, t)-\tau \frac{\partial v(x, y, t)}{\partial t}\right)\right] \\
+d_{21} \nabla^{2} u+d_{22} \nabla^{2} v
\end{gathered}
$$

Expanding (9) in Taylor series and neglecting the higherorder nonlinearities, then (9) becomes

$$
\begin{gathered}
\frac{\partial u}{\partial t}=f(u, v)+d_{11} \nabla^{2} u+d_{12} \nabla^{2} v \\
\frac{\partial v}{\partial t}=v\left(d-h(u, v)+\tau h_{u}(u, v) \frac{\partial u(x, y, t)}{\partial t}\right. \\
\left.+\tau h_{v}(u, v) \frac{\partial v(x, y, t)}{\partial t}\right) \\
+d_{21} \nabla^{2} u+d_{22} \nabla^{2} v
\end{gathered}
$$

where $h_{u}(u, v)=(\partial h(u, v) / \partial u)$ and $h_{v}(u, v)=(\partial h(u, v) / \partial v)$.
From (10), we finally obtain that

$$
\begin{gathered}
\frac{\partial u}{\partial t}=f(u, v)+d_{11} \nabla^{2} u+d_{12} \nabla^{2} v \\
\frac{\partial v}{\partial t}=\frac{1}{1-v \tau h_{v}(u, v)} \\
\times\left(g(u, v)+v \tau h_{u}(u, v) f(u, v)\right. \\
+\left[v \tau h_{u}(u, v) d_{11}+d_{21}\right] \nabla^{2} u \\
\left.+\left[v \tau h_{u}(u, v) d_{12}+d_{22}\right] \nabla^{2} v\right) .
\end{gathered}
$$

Equation (11) can be used to analyze the bifurcation of model (4) when $\tau$ is small.

It is easy to obtain that model (11) has three boundary equilibria $E_{1}=(0,0), E_{2}=((a / r), 0)$, and $E_{3}=(0,(b d / r))$ and a unique positive equilibrium $E^{*}=\left(u^{*}, v^{*}\right)=(((d m-$ $\left.d+c r) / a c),\left(((c a b+(d m-d+c r)(1-m)) d) / a c^{2}\right)\right)$.

Remark 1. In the case without time delay and cross-diffusion, that is, $\tau=0$ and $d_{12}=d_{21}=0$, model (11) becomes (2). It is easy to see that $E_{1}, E_{2}, E_{3}$, and $E^{*}$ are also equilibria of model (2). The stabilities of these equilibria of model (2) can be seen in [21].

In the following, we are mainly concerned with the properties of Hopf and Turing bifurcations for model (11).

Let $0=\mu_{0}<\mu_{1}<\mu_{2}<\cdots$ be the eigenvalues of the operator $-\Delta$ on $\Omega$ with the zero-flux boundary conditions. And setting

$$
\mathbf{X}=\left\{(u, v) \in\left[H^{2}(\Omega)\right]^{2} \mid \partial_{n} u=\partial_{n} v=0 \text { on } \partial \Omega\right\}
$$

$E(\mu)$

$$
=\left\{\phi \mid-\Delta \phi=\mu \phi \text { in } \Omega, \partial_{\nu} \phi=0 \text { on } \partial \Omega\right\}, \quad \text { with } \mu \in \mathbb{R}^{1},
$$

$\left\{\phi_{i j} \mid j=1, \ldots, \operatorname{dim} E\left(\mu_{i}\right)\right\}$ will be an orthonormal basis of $E\left(\mu_{i}\right)$, and $\mathbf{X}_{i j}=\left\{\mathbf{c} \phi_{i j} \mid \mathbf{c} \in \mathbb{R}^{2}\right\}$; then $\mathbf{X}=\bigoplus_{i=1}^{\infty} \mathbf{X}_{i}$, where $\mathbf{X}_{i}=\bigoplus_{j=1}^{\operatorname{dim} E\left(\mu_{i}\right)} \mathbf{X}_{i j}$.

The linearization of model (11) around $E^{*}=\left(u^{*}, v^{*}\right)$ can be expressed by

$$
\frac{\partial}{\partial t}\left(\begin{array}{l}
u \\
v
\end{array}\right)=J_{E^{*}}\left(\begin{array}{l}
u \\
v
\end{array}\right)+\widetilde{D} \nabla^{2}\left(\begin{array}{l}
u \\
v
\end{array}\right),
$$

with domain $\mathbf{X}$. Here,

$$
\begin{aligned}
J_{E^{*}} & =\left(\begin{array}{cc}
f_{u} & f_{v} \\
\mathscr{X}\left(g_{u}+v^{*} \tau h_{u} f_{u}\right) & \mathscr{X}\left(g_{v}+v^{*} \tau h_{u} f_{v}\right)
\end{array}\right) \\
& \triangleq\left(\begin{array}{ll}
J_{11} & J_{12} \\
J_{21} & J_{22}
\end{array}\right), \\
\widetilde{D} & =\left(\begin{array}{cc}
d_{11} \\
\mathscr{X}\left(v^{*} \tau h_{u} d_{11}+d_{21}\right) & \mathscr{X}\left(v^{*} \tau h_{u} d_{12}+d_{22}\right)
\end{array}\right),
\end{aligned}
$$


with $f_{u}=\left.(\partial f / \partial u)\right|_{\left(u^{*}, v^{*}\right)}, f_{v}=\left.(\partial f / \partial v)\right|_{\left(u^{*}, v^{*}\right)}, g_{u}=$ $\left.(\partial g / \partial u)\right|_{\left(u^{*}, v^{*}\right)}, g_{v}=\left.(\partial g / \partial v)\right|_{\left(u^{*}, v^{*}\right)}, h_{u}=\left.(\partial h / \partial u)\right|_{\left(u^{*}, v^{*}\right)}, h_{v}=$ $\left.(\partial h / \partial v)\right|_{\left(u^{*}, v^{*}\right)}$, and $\left(1 /\left.\left(1-v \tau h_{v}\right)\right|_{\left(u^{*}, v^{*}\right)}\right)=(1 /(1-\tau d)) \triangleq \mathscr{X}$. Since $\tau$ is small, we only consider this case of $\tau<(1 / d)$ (i.e. $x>0)$ in this paper.

For each $j(j=0,1,2, \ldots), \mathbf{X}_{j}$ is invariant under the operator $\widetilde{D} \nabla^{2}+L_{E^{*}}$, and $\lambda$ is an eigenvalue of $D \nabla^{2}+L_{E}$ on $\mathbf{X}_{j}$ if and only if $\lambda$ is an eigenvalue of the matrix $M_{j}=-\widetilde{D} \mu_{j}+J_{E^{*}}$ for some $j \geq 0$.

So the stability is reduced to consider the characteristic equation

$$
\operatorname{det}\left(\lambda I-M_{j}\right)=\lambda^{2}-\operatorname{trace}\left(M_{j}\right) \lambda+\operatorname{det}\left(M_{j}\right)=0,
$$

where

$$
\begin{aligned}
\operatorname{trace}( & \left.M_{j}\right)=\operatorname{trace}(\widetilde{D}) \mu_{j}-\operatorname{trace}\left(J_{E^{*}}\right), \\
\operatorname{det}\left(M_{j}\right)= & \mathscr{X} \operatorname{det}(D) \mu_{j}^{2} \\
& -\mathscr{X}\left(d_{11} g_{v}-d_{12} g_{u}-d_{21} f_{v}+d_{22 f_{u}}\right) \mu_{j} \\
& +\operatorname{det}\left(J_{E^{*}}\right) .
\end{aligned}
$$

2.1. Hopf Bifurcation. In this subsection, we analyze the properties of Hopf bifurcation for model (11). For Hopf bifurcation to occur at the positive equilibrium $E^{*}$, the matrix $M_{j}=-\widetilde{D} \mu_{j}+J_{E^{*}}$ must have an eigenvalues on the imaginary axis [52]; that is, $\operatorname{trace}\left(M_{j}\right) \equiv 0$. Therefore, the only possible critical values of $\tau$ are $\tau(i)$ such that, for $i \geq 0$,

$$
\begin{aligned}
\operatorname{trace}(\widetilde{D}) \mu_{i}-\operatorname{trace}\left(J_{E^{*}}\right) \\
=\operatorname{trace}(\widetilde{D}) \mu_{i}-f_{u} \\
\quad+\left(g_{v}+v^{*} \tau(i) h_{u} f_{v}\right) \mathscr{X}=0 .
\end{aligned}
$$

At $\tau=\tau(i)$, $\operatorname{trace}\left(M_{i}\right)=\operatorname{trace}\left(\widetilde{D}\left(\mu_{i}-\mu_{j}\right)\right)$, and therefore, if $i \geq 1$, for all $0 \leq j<i$, $\operatorname{trace}\left(M_{i}\right)>0$ and the matrix $M_{j}=-\widetilde{D} \mu_{j}+J_{E^{*}}$ has at least $2 i$ eigenvalues with positive real parts. Therefore, the only value of $\tau$ at which Hopf bifurcation hypotheses may be satisfied is

$$
\tau_{c}=\tau(0):=\frac{f_{u}+g_{v}}{v^{*}\left(f_{u} h_{v}-h_{u} f_{v}\right)} .
$$

Near $\tau_{c}$, the complex conjugate pair $\kappa(\tau) \pm i \omega(\tau)$ is given by

$$
\kappa(\tau)=\frac{1}{2} \operatorname{trace}\left(J_{E^{*}}\right), \quad \omega^{2}(\tau)=\operatorname{det}\left(J_{E^{*}}\right)-\kappa^{2}(\tau) .
$$

Since $\kappa\left(\tau_{c}\right)=0$ and $\operatorname{det}\left(J_{E^{*}}\right)=(((d m+r c-d) d) / c) \mathscr{X}>0$, there exists an interval $I$ containing $\tau_{c}$ such that

(i) $\omega^{2}(\tau) \neq 0$ for all $\tau \in I$;

(ii) $\operatorname{trace}\left(M_{j}\right)<0$ and $\operatorname{det}\left(M_{j}\right)>0$ with $d_{11} g_{v}-d_{12} g_{u}-$ $d_{21} f_{v}+d_{22 f_{u}}<0$ uniformly for $j \geq 1$ and $\tau \in I$;

(iii) $\left.(d / d \tau) \kappa(\tau)\right|_{\tau=\tau_{c}}=v^{*} f_{v} h_{u}+\left(d g_{v} /\left(1-d \tau_{c}\right)^{2}\right)>0$.
Thus, we have the following theorem.

Theorem 2. If $d_{11} g_{v}-d_{12} g_{u}-d_{21} f_{v}+d_{22 f_{u}}<0$, then model (11) undergoes a Hopf bifurcation that occurs around the positive equilibrium $E^{*}$ at $\tau=\tau_{c}$.

2.2. Turing Instability. In this subsection, we will state the Turing instability for the positive equilibrium $E^{*}$ of model (11).

Mathematically speaking, the positive equilibrium $E^{*}$ is Turing instability, which was emphasized by Turing in his pioneering work in 1952 [13], which means that it is asymptotically stable in the absence of diffusion in model (11) but unstable with respect to solutions in model (11). Hence, Turing instability occurs when either the condition $\operatorname{trace}\left(M_{j}\right)<0$ or the condition $\operatorname{det}\left(M_{j}\right)>0$ is violated.

Since the positive equilibrium $E^{*}$ is stable without diffusion which means that $\operatorname{trace}\left(J_{E^{*}}\right)<0$ and $\operatorname{det}\left(J_{E^{*}}\right)>0$ hold, then $\operatorname{trace}\left(M_{j}\right)<0$ is always true. Therefore, for the emergency of the diffusion-driven instability in model (11), it is needed that $\operatorname{det}\left(M_{j}\right)<0$ for some $\mu_{j}>0$. A necessary condition is

$$
d_{11} g_{v}-d_{12} g_{u}-d_{21} f_{v}+d_{22 f_{u}}>0
$$

otherwise $\operatorname{det}\left(M_{j}\right)>0$ for all $j>0$ since $\mathscr{X} \operatorname{det}(D)>0$ and $\operatorname{det}\left(J_{E^{*}}\right)>0$. And we notice that $\operatorname{det}\left(M_{j}\right)$ achieves its minimum:

$$
\begin{aligned}
\min _{j \in \mathbf{R}^{+}} \operatorname{det} & \left(M_{j}\right) \\
=(( & 4 \mathscr{X} \operatorname{det}(D) \operatorname{det}\left(J_{E^{*}}\right) \\
& \left.-\mathscr{X}\left(d_{11} g_{v}-d_{12} g_{u}-d_{21} f_{v}+d_{22 f_{u}}\right)^{2}\right) \\
& \left.\times(4 \operatorname{det}(\widetilde{D}))^{-1}\right)
\end{aligned}
$$

at the critical value $\mu_{c}^{2}>0$ where

$$
\mu_{c}^{2}=\frac{d_{11} g_{v}-d_{12} g_{u}-d_{21} f_{v}+d_{22 f_{u}}}{2 \operatorname{det}(D)} .
$$

Summarizing the above calculation, we conclude the following.

Theorem 3. If

$$
\tau<\min \left\{\frac{1}{d}, \tau_{c}\right\},
$$

$4 \mathscr{X} \operatorname{det}(D) \operatorname{det}\left(J_{E^{*}}\right)$

$$
\begin{aligned}
& -\mathscr{X}\left(d_{11} g_{v}-d_{12} g_{u}-d_{21} f_{v}+d_{22 f_{u}}\right)^{2}<0, \\
& d_{11} g_{v}-d_{12} g_{u}-d_{21} f_{v}+d_{22 f_{u}}>0,
\end{aligned}
$$

then the positive equilibrium $E^{*}$ of model (11) is Turing unstable. 


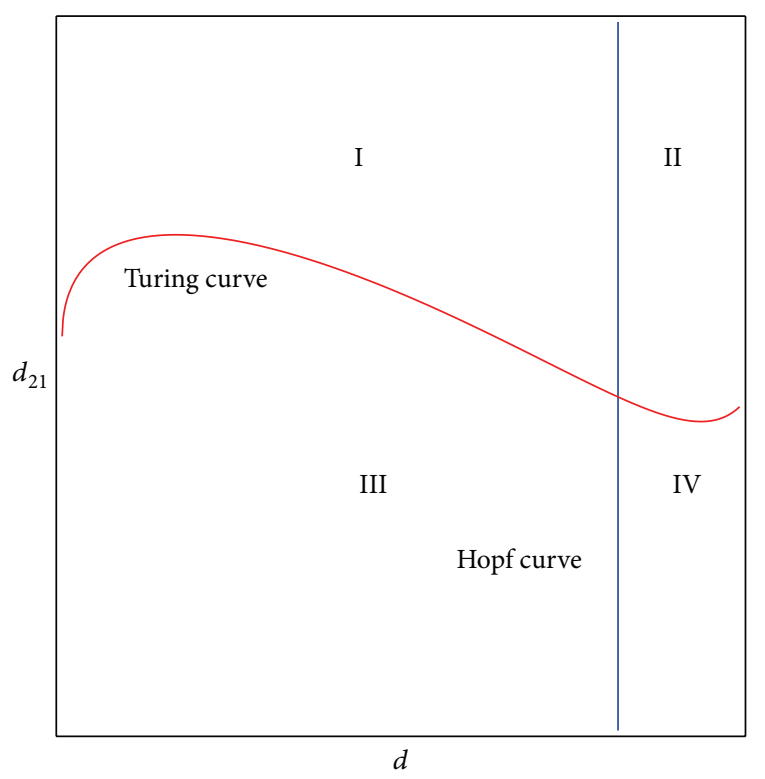

Figure 1: Bifurcation diagram for model (11) in the $d-d_{21}$ parameter space. The red line is Turing bifurcation curve, and the blue line denotes Hopf bifurcation. Domain I is the region of pure Turing instability. In domain II, Hopf-Turing instability occurs. And domain III is the stable region. Domain IV is the region of pure Hopf instability.

In Figure 1, we show the bifurcation diagram for model (11). The Turing instability curve and Hopf bifurcation curve separate the $d-d_{21}$ parameter space into four domains. The domain on the left of the Hopf bifurcation curve is stable; the domain above Turing bifurcation curve is unstable. Hence, among these domains, only the domain I satisfies conditions of Theorem 3, and we call domain I as Turing space, where the Turing instability occurs and the Turing patterns may be undergone. Domain IV gives birth to spiral patterns.

\section{Pattern Formation via Numerical Simulations}

In this section, we perform extensive numerical simulations of the spatially extended model (4) in two-dimension spaces, and the qualitative results are shown here. Our numerical simulations employ a system size of $100 \times 100$ space units. And some parameters are taken as

$$
\begin{aligned}
& b=0.22, \quad c=0.4, \quad m=0.17, \\
& d_{12}=0.05, \quad \tau=0.46 .
\end{aligned}
$$

The numerical integration of model (4) was performed by means of forward Euler integration, with a time step of $\Delta t=$ 0.001 and a space step of $\Delta x=\Delta y=(1 / 4)$, using the standard five-point approximation for the $2 \mathrm{D}$ Laplacian with the zeroflux boundary conditions [53].

3.1. Turing Pattern Formation in Domain I. In this subsection, we first consider the pattern formation when parameters are located in domain I of Figure 1, which is pure Turing unstable domain.

Initially, the entire system is placed in the steady state $\left(u^{*}, v^{*}\right)$, and the propagation velocity of the initial perturbation is thus on the order of $5 \times 10^{-4}$ space units per time unit. The initial data of prey $u$ is a $101 \times 101$ matrix, such as

$$
\begin{aligned}
& u\left(x, y, t_{0}\right) \\
& =\left(\begin{array}{cccc}
u^{*} & u^{*} & \cdots & u^{*} \\
u^{*} & u^{*} & \cdots & u^{*} \\
\vdots & \vdots & \cdots & \vdots \\
u^{*} & u^{*} & \cdots & u^{*}
\end{array}\right) \\
& +(\operatorname{rand}(101,101)-0.5) \times 10^{-3}, \quad t_{0} \in[-\tau, 0],
\end{aligned}
$$

where $\operatorname{rand}(101,101)$ is MATLAB procedure, which returns a $101 \times 101$ matrix containing pseudorandom values drawn from the standard uniform distribution on the open interval $(0,1)$. And the system is then integrated for 2,000,000 time steps, and the last images are saved. After the initial period during which the perturbation spreads, the system goes into an essentially steady state (time independent).

In the numerical simulations, different types of dynamics are observed, and it is found that the distributions of predator and prey are always of the same type. Consequently, we can restrict our analysis of pattern formation to one distribution. In the following, we show the distribution of prey $u$.

In Figure 2, we show three typical Turing patterns and their three-dimension pattern for model (4) at $t=2000$. In the case of $\left(d, d_{21}, d_{22}\right)=(0.08,0.89,0.37)$, the equilibrium is $\left(u^{*}, v^{*}\right)=(2.8697,0.5203)$ and the holes patterns are isolated zones with low prey density (cf. Figure 2(a)). When increasing $d$ to $0.4,\left(d, d_{21}, d_{22}\right)=(0.47,0.89,0.37),\left(u^{*}, v^{*}\right)=$ $(0.9877,1.2218)$, the stripes patterns appear. While with $\left(d, d_{21}, d_{22}\right)=(0.61,0.39,0.75),\left(u^{*}, v^{*}\right)=(0.3122,0.7306)$, spots patterns are isolated zones with high prey density (cf. Figure 2(c)). And Figures 2(d)-2(e) are three-dimension patterns corresponding to Figures $2(\mathrm{a})-2(\mathrm{c})$.

On the other hand, it is easy to check that holes, stripes, and spots also appear in model (4) without time delay for $\tau=0$ and other the parameters are taken the same way as in Figures 2(a)-2(c), respectively. However, in parameters of Figure $2, \operatorname{trace}\left(M_{j}\right)<0$ and $\operatorname{det}\left(M_{j}\right)>0$ for all $j \geq 0$ if the cross-diffusion coefficients $d_{12}=0$ and $d_{21}=0$. That is to say, model (4) is stable in absence of cross-diffusion, and this instability is induced by cross-diffusion.

3.2. Chaotic Pattern Formation Domain II. In this subsection, we will focus on the pattern formation when parameters are located in domain II of Figure 1, the domain of pure HopfTuring instability.

Figure 3 shows the evolution process of the spatial pattern of $u$ at time $t=0,100,1000,2000$ with parameter set (25) and $a=0.1, b=0.71, r=1.54, d=0.47$, $d_{11}=0.2, d_{21}=0.44, d_{22}=0.88$, and $\tau=0.46$. The pattern takes a long time to settle down; starting with 


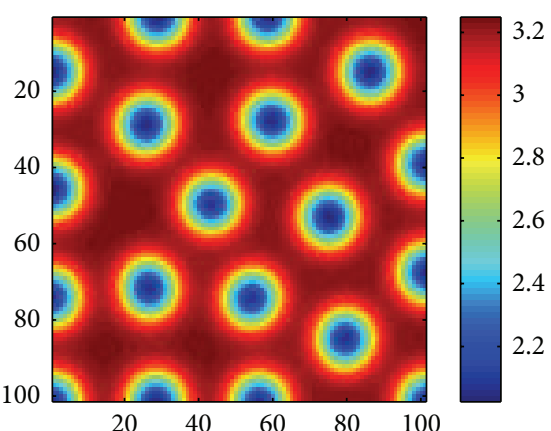

(a)

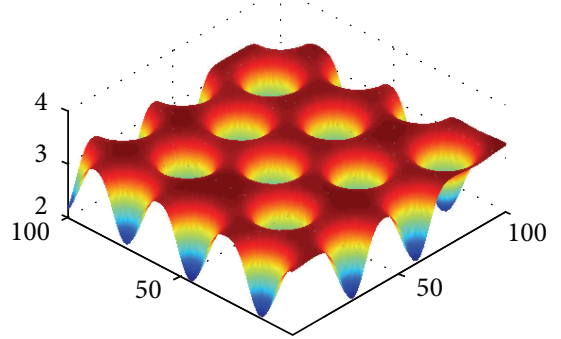

(d)

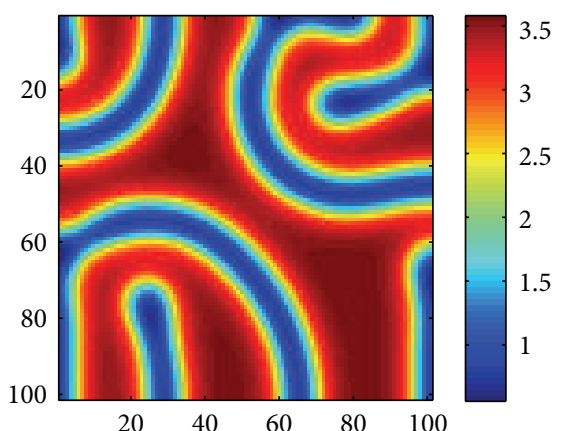

(b)

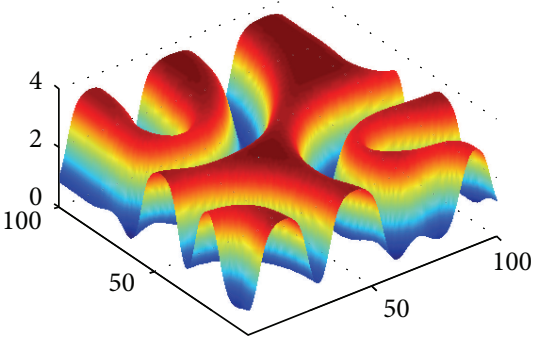

(e)

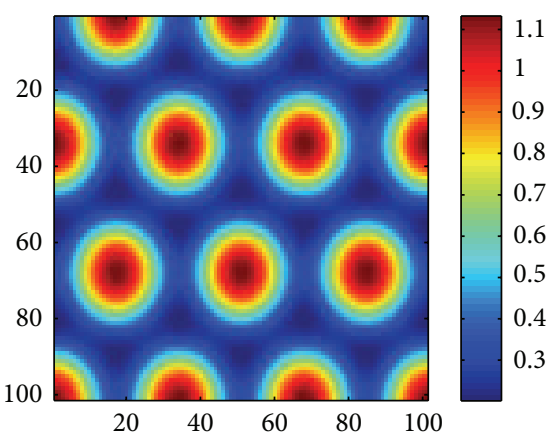

(c)

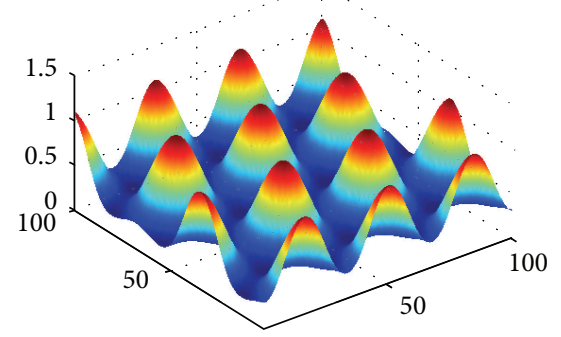

(f)

FIGURE 2: Stationary Turing patterns of $u$ in model (4) at time $t=2000$ with parameters $a=0.43, b=0.22, c=0.4, r=1.4, m=0.17, d_{11}=$ $0.4, d_{12}=0.05, d_{22}=0.37, \tau=0.46$, and (a) (d) $\left(d, d_{21}, d_{22}\right)=(0.08,0.89,0.37)$, (b) (e) $\left(d, d_{21}, d_{22}\right)=(0.4,0.89,0.37)$, and (c) (f) $\left(d, d_{21}, d_{22}\right)=$ $(0.61,0.39,0.75)$, and the other parameters are fixed as (25). Times: $t=2000$.

a homogeneous state $E^{*}=(56.4750,55.3357)$ (cf. Figure 3(a)), the random initial distribution leads to the formation of a strongly irregular transient pattern in the domain. After the irregular pattern form (cf. Figure 3(b)), it grows slightly and "jumps" alternately for a certain time, and finally the chaos patterns prevail over the whole domain (cf. Figures 3(c)-3(d)), which is time-dependent. For the sake of learning the spatiotemporal dynamics of model (4) further, we illustrate the time-series plots and phase portraits in Figures 3(e)-3(f). From the time-series plots in Figure 3(e), one can see that $u$ and $v$ strongly oscillates over time $t$. And in Figure 3(f), there exhibits a "local" phase plane of the system invaded by the irregular spatiotemporal oscillations; the trajectory now fills nearly the whole domain inside the quasilimit cycle. This regime of the system dynamics corresponds to spatiotemporal chaos. And the spatial symmetry of model (4) is broken, and the patterns are oscillatory in space.

3.3. Spiral Wave Pattern Formation Domain IV. In this subsection, we will focus on the pattern formation when parameters are located in domain IV of Figure 1, the domain of pure Hopf instability.

In order to make any influence of the corners of the domain more visible, and thanks to the insightful work of Medvinsky et al. [4] and Upadhyay et al. [54], we have studied the spiral wave pattern for the following initial condition (27). In this case, we employ $\Delta x=\Delta y=1$ and the system size is $200 \times 200$.
In order to make any influence of the corner of the domain more visible, following $[43,55]$, the initial conditions are deliberately chosen to be unsymmetrical as

$$
\begin{aligned}
u\left(x, y, t_{0}\right) & =u^{*}-\varepsilon_{1}(x-100), \\
v\left(x, y, t_{0}\right) & =v^{*}-\varepsilon_{2}(y-100), \\
t_{0} & \in[-\tau, 0]
\end{aligned}
$$

where $\varepsilon_{1}=2 \times 10^{-4}$ and $\varepsilon_{2}=3 \times 10^{-4}$. For more clarity, we put the initial data of prey $u$ as follow

$$
\begin{aligned}
& u\left(x, y, t_{0}\right) \\
& =\left(\begin{array}{cccc}
u^{*}+\varepsilon_{1}(100-0) & u^{*}+\varepsilon_{1}(100-0) & \cdots & u^{*}+\varepsilon_{1}(100-0) \\
u^{*}+\varepsilon_{1}(100-1) & u^{*}+\varepsilon_{1}(100-1) & \cdots & u^{*}+\varepsilon_{1}(100-1) \\
\vdots & \vdots & \cdots & \vdots \\
u^{*}+\varepsilon_{1}(100-200) & u^{*}+\varepsilon_{1}(100-200) & \cdots & u^{*}+\varepsilon_{1}(100-200)
\end{array}\right), \\
& t_{0} \in[-\tau, 0] .
\end{aligned}
$$

Figure 4 shows the evolution process of the spatial pattern of prey $u$ at $t=0,500,1500,3000$ with $\left(d, d_{21}, d_{22}\right)=$ $(0.5,0.15,0.37)$; the other parameters are fixed as in $(25)$. Figure 4(a) shows that, for the model (4) with initial conditions (27), the formation of the irregular patchy structure can be preceded by the evolution of a regular spiral spatial pattern. Note that the appearance of the spirals is not induced by the initial conditions. The center of each spiral is situated in a critical point $\left(x_{\mathrm{cr}}, y_{\mathrm{cr}}\right)=(100,100)$, where $u\left(x_{\mathrm{cr}}, y_{\mathrm{cr}}\right)=$ $u^{*}, v\left(x_{\mathrm{cr}}, y_{\mathrm{cr}}\right)=v^{*}$. After the spirals form (Figure 4(b)), they 


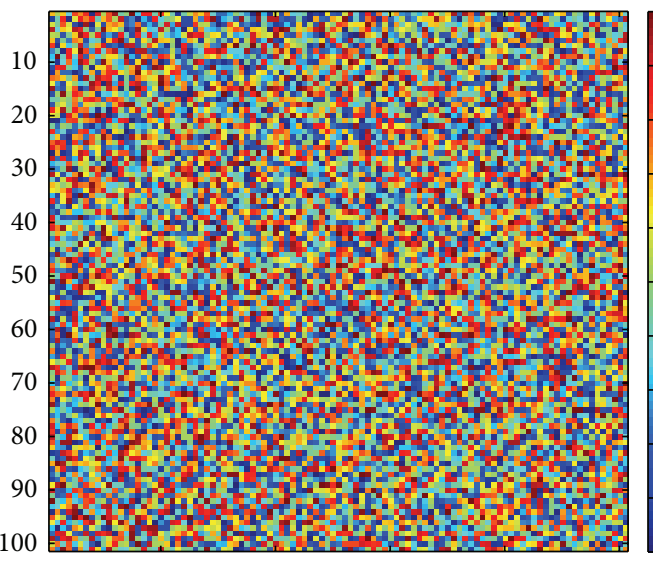

20

40

60

(a)

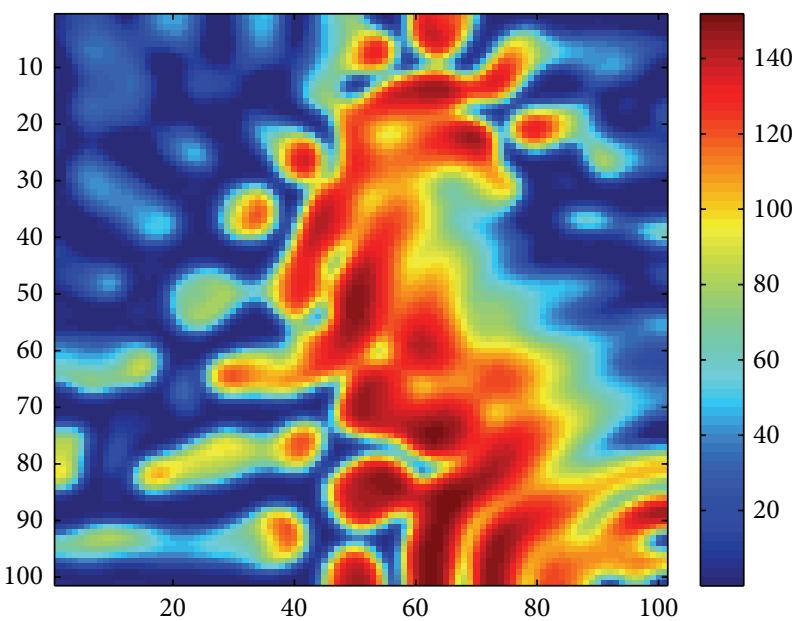

(c)

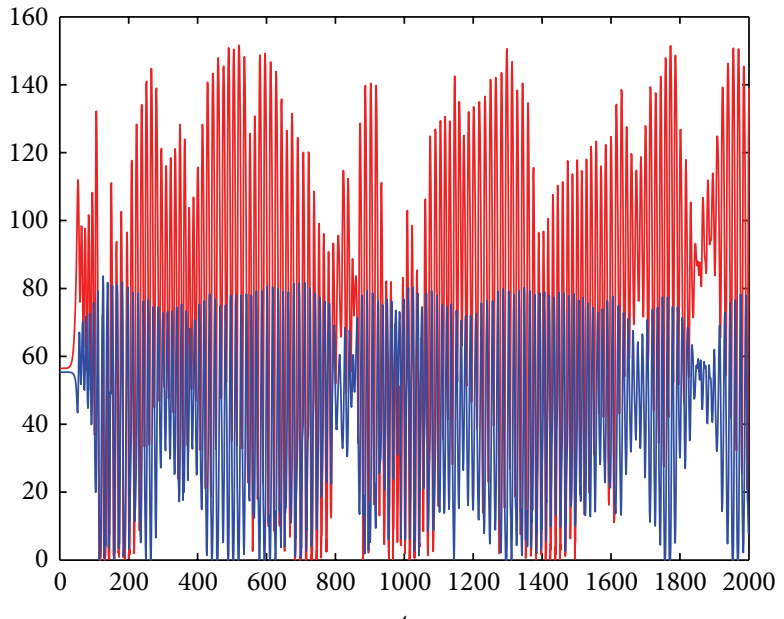

(e)

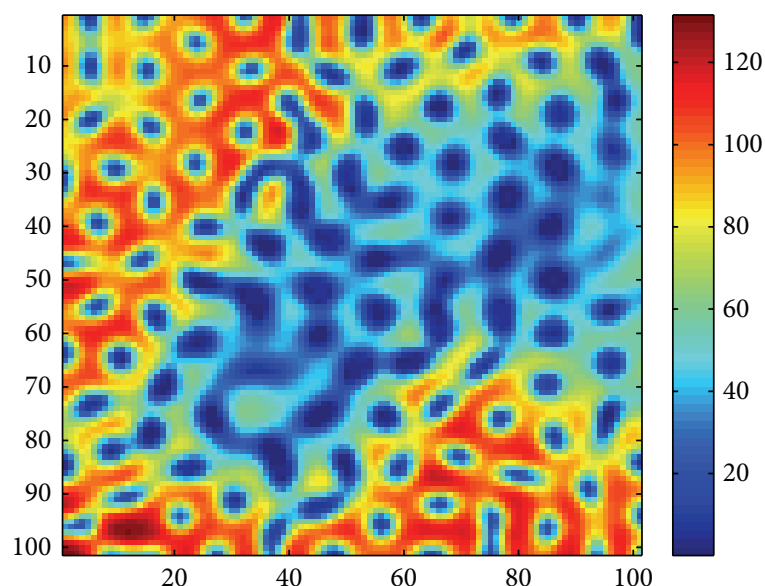

(b)

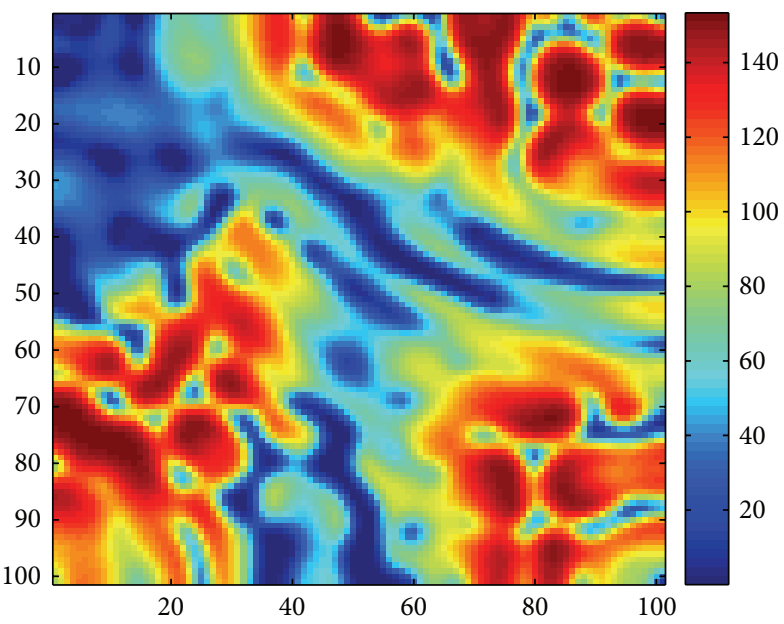

(d)

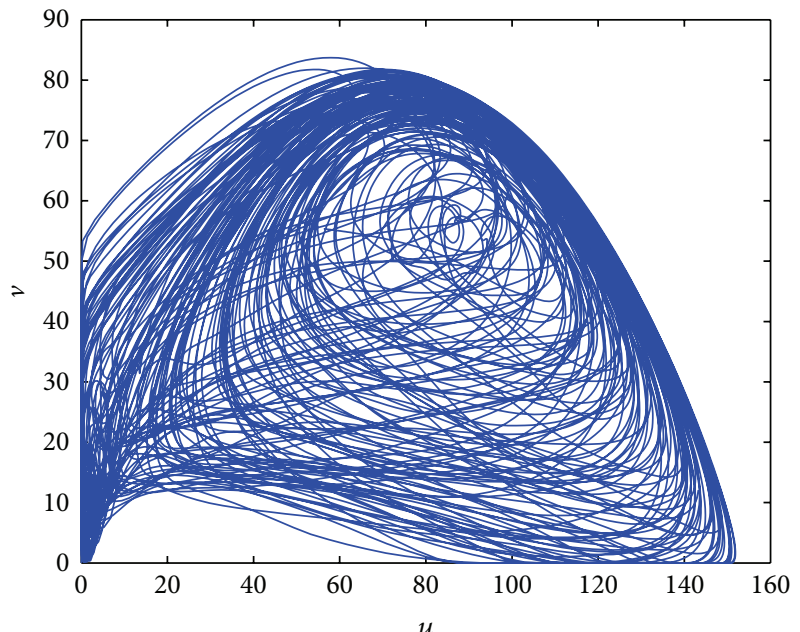

(f)

FIGURE 3: Chaotic patterns of $u$ in model (4) with parameters $a=0.01, b=0.22, c=0.4, r=1.54, m=0.17, d_{11}=0.2, d_{12}=0.05, d_{21}=$ $0.44, d_{22}=0.88$, and $\tau=0.46$. Times: (a) $t=0$, (b) $t=100$, (c) $t=1000$, (d) $t=2000$, (e) time-series plots, and (f) phase portraits. 


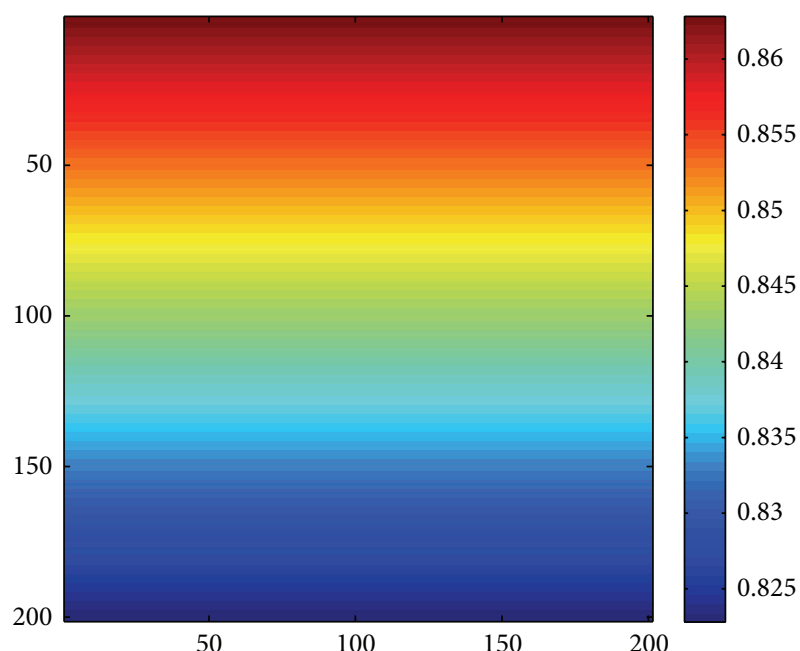

(a)

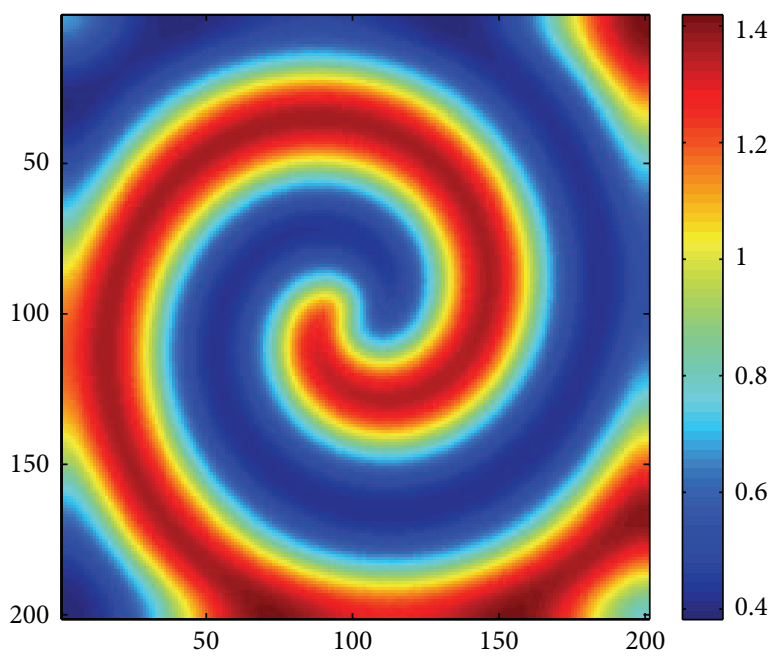

(c)

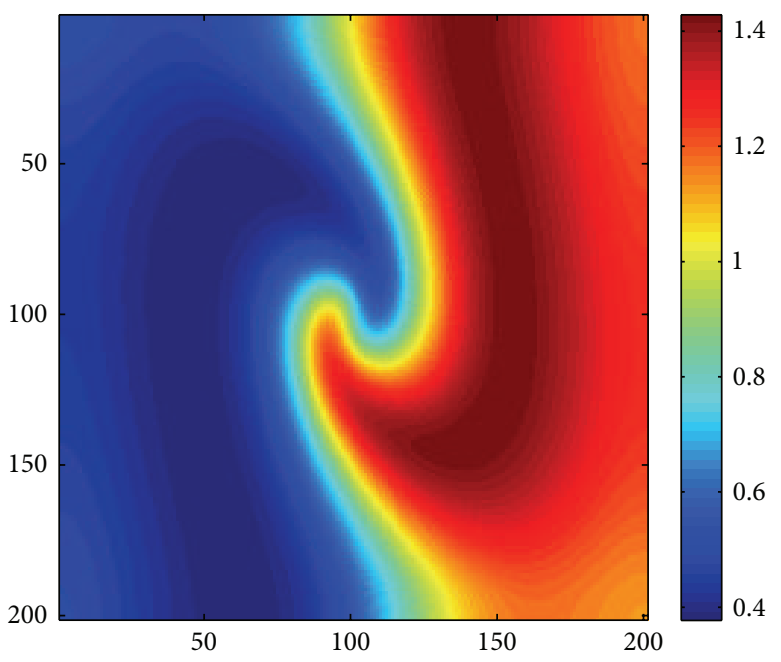

(b)

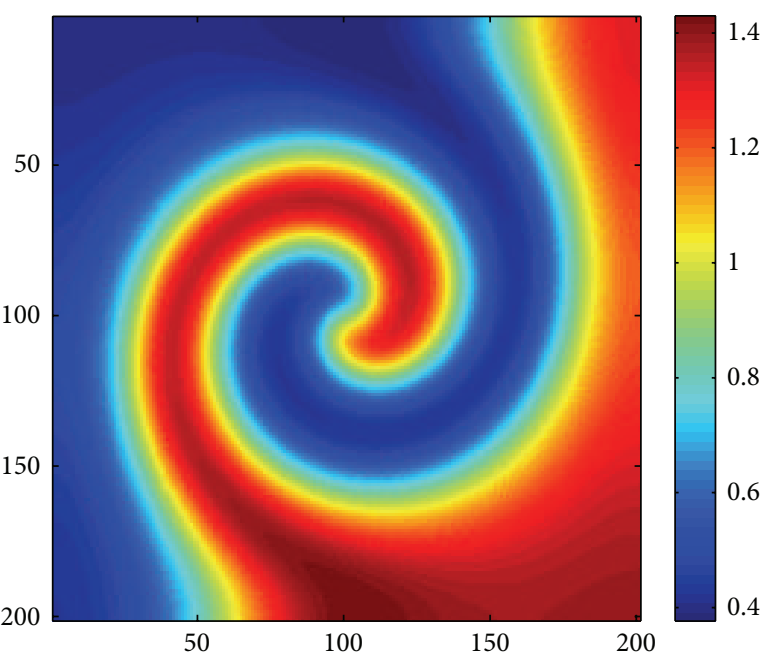

(d)

FIGURE 4: The process of formation of spiral pattern of $u$ in model (4) with parameters $a=0.43, b=0.22, c=0.4, r=1.4, m=0.17, d=0.5$, $d_{11}=0.4, d_{12}=0.05, d_{21}=0.15, d_{22}=0.37$, and $\tau=0.46$. Times: (a) $t=0$, (b) $t=500$, (c) $t=1500$, and (d) $t=3000$.

grow slightly for a certain time and their spatial structure becomes more distinct (Figures 4(c) and 4(d)). Obviously, spiral pattern arises from Hopf instability.

\section{Concluding Remarks}

In this paper, we analyze the spatiotemporal dynamics of a spatial Leslie-Gower predator-prey model with time delay and diffusion under the zero-flux boundary conditions. The value of this study is twofold. First, it gives the conditions of Hopf and Turing instability induced by the effects of time delay and cross-diffusion. Second, it gives three domains for three kinds of patterns of model (4) and finds that time delay and cross-diffusion may induce Turing instability, resulting in stationary Turing patterns, Hopf instability, resulting in spiral wave patterns, and Turing-Hopf instability, resulting in chaotic wave patterns.
If $\tau$ is small enough, it is worthy to note that, for the delayed model (4), two typical patterns are similar to those in our previous work [43] (a special case of model (4) with self diffusion): pure Turing instability gives birth to the holes, stripes, and spots patterns (cf. Figure 2) and pure Hopf instability to spiral wave pattern (cf. Figure 4). But this is not forever. In the present paper, we find that the delayed reaction-diffusion model (4) exhibits more complex pattern formation, for there exists the third pattern, chaotic wave pattern, which is induced by Turing-Hopf instability. The complexity of the pattern formation is induced by the crossdiffusion effect.

The results of the formation of these patterns may indicate the vital role of phase transience regimes in the spatiotemporal organization of the prey and predator in the real world situation. It is also important to distinguish between "intrinsic" patterns, that is, Turing patterns arising due to predation, and "forced" patterns induced by the heterogeneity 
of the environment, that is, spiral wave pattern. Because of the environmental heterogeneity, the dispersion of varying quantities and typical times and lengths can be essentially different in different cases and thus can induce different spatial patterns.

\section{Conflict of Interests}

The authors declare that there is no conflict of interests regarding the publication of this paper.

\section{Acknowledgments}

The authors would like to thank the anonymous referee for the very helpful suggestions and comments which led to the improvement of our original paper. This research was supported by the National Science Foundation of China (61373005), Zhejiang Provincial Natural Science Foundation (LY12A01014), and the Fund Project of Zhejiang Provincial Education Department (Y201223449).

\section{References}

[1] C. Neuhauser, "Mathematical challenges in spatial ecology," Notices of the American Mathematical Society, vol. 48, no. 11, pp. 1304-1314, 2001.

[2] R. S. Cantrell and C. Cosner, Spatial Ecology via ReactionDiffusion Equations, John Wiley \& Sons, 2003.

[3] G. Nicolis and I. Prigogine, Self-Organization in Nonequibibrium System, John Wiley \& Sons, 1997.

[4] A. B. Medvinsky, S. V. Petrovskii, I. A. Tikhonova, H. Malchow, and B.-L. Li, "Spatiotemporal complexity of plankton and fish dynamics," SIAM Review, vol. 44, no. 3, pp. 311-370, 2002.

[5] J. D. Murray, "Discussion: turing's theory of morphogenesis: its influence on modelling biological pattern and form," Bulletin of Mathematical Biology, vol. 52, no. 1-2, pp. 119-152, 1990.

[6] A. Gierer and H. Meinhardt, "A theory of biological pattern formation," Biological Cybernetic, vol. 12, no. 1, pp. 30-39, 1972.

[7] M. C. Cross and P. C. Hohenberg, "Pattern formation outside of equilibrium," Reviews of Modern Physics, vol. 65, no. 3, pp. 851-1112, 1993.

[8] P. Jung and G. Mayer-Kress, "Spatiotemporal stochastic resonance in excitable media," Physical Review Letters, vol. 74, no. 11, pp. 2130-2133, 1995.

[9] Y. N. Kyrychko, K. B. Blyuss, S. J. Hogan, and E. Schöll, "Control of spatiotemporal patterns in the Gray-Scott model," Chaos, vol. 19, no. 4, Article ID 043126, 2009.

[10] K. J. Lee, W. D. McCormick, Q. Ouyang, and H. L. Swinney, "Pattern formation by interacting chemical fronts," Science, vol. 261, no. 5118, pp. 192-194, 1993.

[11] B. Lindner, J. García-Ojalvo, A. Neiman, and L. SchimanskyGeier, "Effects of noise in excitable systems," Physics Reports, vol. 392, no. 6, pp. 321-424, 2004.

[12] H. Malchow and S. V. Petrovskii, "Dynamical stabilization of an unstable equilibrium in chemical and biological systems," Mathematical and Computer Modelling, vol. 36, no. 3, pp. 307319, 2002.

[13] A. M. Turing, "The chemical basis of morphogenesis," Philosophical Transactions of the Royal Society of London B, vol. 237, no. 641 , pp. 37-72, 1952.
[14] G. Nicolis and I. Prigogine, Biological Invasions: Global Perspective, John Wiley \& Sons, 1989.

[15] S. N. Higesada and K. Kawasaki, Biological Invasions: Theory and Practice, Oxford University Press, 1997.

[16] M. A. Aziz-Alaoui and M. Daher Okiye, "Boundedness and global stability for a predator-prey model with modified LeslieGower and Holling-type II schemes," Applied Mathematics Letters, vol. 16, no. 7, pp. 1069-1075, 2003.

[17] M. A. Aziz-Alaoui and M. Daher Okiye, "On the dynamics of a predator-prey model with the holling-tanner functional response," in Proceedings of the 5th European Conference on Mathematical and Theoretical Biology (ESMTB '02), V. Capasso, Ed., pp. 270-278, 2002.

[18] B. I. Camara and M. A. Aziz-Alaoui, "Dynamics of a predatorprey model with diffusion," Dynamics of Continuous, Discrete \& Impulsive Systems A, vol. 15, no. 6, pp. 897-906, 2008.

[19] B. I. Camara and M. A. Aziz-Alaoui, "Turing and Hopf patterns formation in a predator-prey model with Leslie-Gowertype functional response," Dynamics of Continuous, Discrete \& Impulsive Systems B, vol. 16, no. 4, pp. 479-488, 2009.

[20] B. Camara, Complexité de dynamiques de modles proieprédateur avec diffusion et application [Ph.D. thesis], Universit du Havre, 2009.

[21] X. N. Guan, W. M. Wang, and Y. L. Cai, "Spatiotemporal dynamics of a Leslie-Gower predator-prey model incorporating a prey refuge," Nonlinear Analysis: Real World Applications, vol. 12, no. 4, pp. 2385-2395, 2011.

[22] M. Ferrara and L. Guerrini, "An economic growth model with power law logistic technology and time delay," Far East Journal of Mathematical Sciences, vol. 72, no. 1, pp. 169-174, 2013.

[23] C. Bianca, M. Ferrara, and L. Guerrini, "Hopf bifurcations in a delayed-energy-based model of capital accumulation," Applied Mathematics \& Information Sciences, vol. 7, no. 1, pp. 139-143, 2013.

[24] C. Bianca, M. Ferrara, and L. Guerrini, "The Cai model with time delay: existence of periodic solutions and asymptotic analysis," Applied Mathematics \& Information Sciences, vol. 7, no. 1, pp. 21-27, 2013.

[25] M. Ferrara, "A note on convergence speed and population in the AK model with habit formation," International Journal of Pure and Applied Mathematics, vol. 67, no. 3, pp. 327-335, 2011.

[26] M. Ferrara, "Technological diffusion and Benthamite utility in the Ramsey model: an analytical exploration," International Journal of Pure and Applied Mathematics, vol. 67, no. 2, pp. 225236, 2011.

[27] C. Bianca, M. Ferrara, and L. Guerrini, "High-order moments conservation in thermostatted kinetic models," Journal of Global Optimization, pp. 1-16, 2013.

[28] W. M. Wang, Y. L. Cai, Y. N. Zhu, and Z. G. Guo, "Allee-effectinduced instability in a reactiondiffusion predator-prey model," Abstract and Applied Analysis, vol. 2013, Article ID 487810, 10 pages, 2013.

[29] S. Wang, W. Liu, Z. Guo, and W. Wang, "Traveling wave solutions in a reaction-diffusion epidemic model," Abstract and Applied Analysis, vol. 2013, Article ID 216913, 13 pages, 2013.

[30] S. Ruan, "On nonlinear dynamics of predator-prey models with discrete delay," Mathematical Modelling of Natural Phenomena, vol. 4, no. 2, pp. 140-188, 2009.

[31] A. F. Nindjin, M. A. Aziz-Alaoui, and M. Cadivel, "Analysis of a predator-prey model with modified Leslie-Gower and Hollingtype II schemes with time delay," Nonlinear Analysis: Real World Applications, vol. 7, no. 5, pp. 1104-1118, 2006. 
[32] R. Yafia, F. El Adnani, and H. T. Alaoui, "Limit cycle and numerical similations for small and large delays in a predatorprey model with modified Leslie-Gower and Holling-type II schemes," Nonlinear Analysis: Real World Applications, vol. 9, no. 5, pp. 2055-2067, 2008.

[33] R. Xu and L. Chen, "Persistence and stability for a twospecies ratio-dependent predator-prey system with time delay in a two-patch environment," Computers \& Mathematics with Applications, vol. 40, no. 4-5, pp. 577-588, 2000.

[34] Q. S. Li and L. Ji, "Control of turing pattern formation by delayed feedback," Physical Review E, vol. 69, no. 4, Article ID 046205, 2004.

[35] K. P. Hadeler and S. G. Ruan, "Interaction of diffusion and delay," Discrete and Continuous Dynamical Systems B, vol. 8, no. 1, pp. 95-105, 2007.

[36] P. Ghosh, S. Sen, and D. S. Ray, "Nonlinear dynamics of finite perturbation: collapse and revival of spatial patterns," Physical Review E, vol. 79, no. 1, Article ID 016206, 2009.

[37] S. Sen, P. Ghosh, S. S. Riaz, and D. S. Ray, "Time-delay-induced instabilities in reaction-diffusion systems," Physical Review E, vol. 80, no. 4, Article ID 046212, 2009.

[38] P. Ghosh, S. Sen, and D. S. Ray, "Reaction-Cattaneo systems with fluctuating relaxation time," Physical Review E, vol. 81, no. 2, Article ID 026205, 2010.

[39] P. Ghosh, "Control of the Hopf-Turing transition by timedelayed global feedback in a reaction-diffusion system," Physical Review E, vol. 84, no. 1, Article ID 016222, 2011.

[40] P. Ghosh, S. Sen, S. S. Riaz, and D. S. Ray, "Controlling birhythmicity in a self-sustained oscillator by time-delayed feedback," Physical Review E, vol. 83, no. 3, Article ID 036205, 2011.

[41] Y. Zhu, Y. Cai, S. Yan, and W. Wang, "Dynamical analysis of a delayed reaction-diffusion predator-prey system," Abstract and Applied Analysis, vol. 2012, Article ID 323186, 23 pages, 2012.

[42] S. Yan, X. Lian, W. Wang, and Y. Wang, "Bifurcation analysis in a delayed diffusive Leslie-Gower model," Discrete Dynamics in Nature and Society, vol. 2013, Article ID 170501, 11 pages, 2013.

[43] X. Z. Lian, H. L. Wang, and W. M. Wang, "Delay-driven pattern formation in a reaction-diffusion predator-prey model incorporating a prey refuge," Journal of Statistical Mechanics, vol. 2013, no. 4, Article ID P04006, 2013.

[44] S. L. Yan, X. Z. Lian, W. M. Wang, and R. K. Upadhyay, "Spatiotemporal dynamics in a delayed diffusive predator model," Applied Mathematics and Computation, vol. 224, pp. 524-534, 2013.

[45] W. Wang, Y. Lin, L. Zhang, F. Rao, and Y. Tan, "Complex patterns in a predator-prey model with self and cross-diffusion," Communications in Nonlinear Science and Numerical Simulation, vol. 16, no. 4, pp. 2006-2015, 2011.

[46] W.-M. Wang, W.-J. Wang, Y.-Z. Lin, and Y.-J. Tan, "Pattern selection in a predation model with self and cross diffusion," Chinese Physics B, vol. 20, no. 3, Article ID 034702, 2011.

[47] V. N. Biktashev, J. Brindley, A. V. Holden, and M. A. Tsyganov, "Pursuit-evasion predator-prey waves in two spatial dimensions," Chaos, vol. 14, no. 4, pp. 988-994, 2004.

[48] J. B. Shukla and S. Verma, "Effects of convective and dispersive interactions on the stability of two species," Bulletin of Mathematical Biology, vol. 43, no. 5, pp. 593-610, 1981.

[49] X. Lian, Y. Yue, and H. Wang, "Pattern formation in a cross-diffusive ratio-dependent predator-prey model," Discrete Dynamics in Nature and Society, vol. 2012, Article ID 814069, 13 pages, 2012.
[50] E. H. Kerner, "Further considerations on the statistical mechanics of biological associations," Bulletin of Mathematical Biophysics, vol. 21, pp. 217-255, 1959.

[51] N. Shigesada, K. Kawasaki, and E. Teramoto, "Spatial segregation of interacting species," Journal of Theoretical Biology, vol. 79, no. 1, pp. 83-99, 1979.

[52] Z. Mei, Numerical Bifurcation Analysis for Reaction-Diffusion Equations, vol. 28, Springer, Berlin, Germany, 2000.

[53] M. R. Garvie, "Finite-difference schemes for reaction-diffusion equations modeling predator-prey interactions in MATLAB," Bulletin of Mathematical Biology, vol. 69, no. 3, pp. 931-956, 2007.

[54] R. K. Upadhyay, W. Wang, and N. K. Thakur, "Spatiotemporal dynamics in a spatial plankton system," Mathematical Modelling of Natural Phenomena, vol. 5, no. 5, pp. 102-122, 2010.

[55] W. M. Wang, L. Zhang, H. L. Wang, and Z. Q. Li, "Pattern formation of a predator-prey system with Ivlev-type functional response," Ecological Modelling, vol. 221, no. 2, pp. 131-140, 2010. 


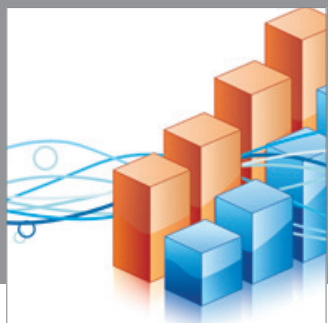

Advances in

Operations Research

mansans

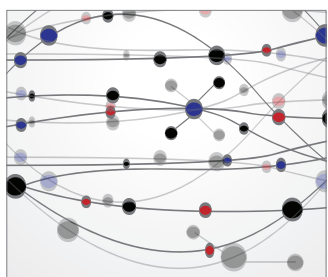

The Scientific World Journal
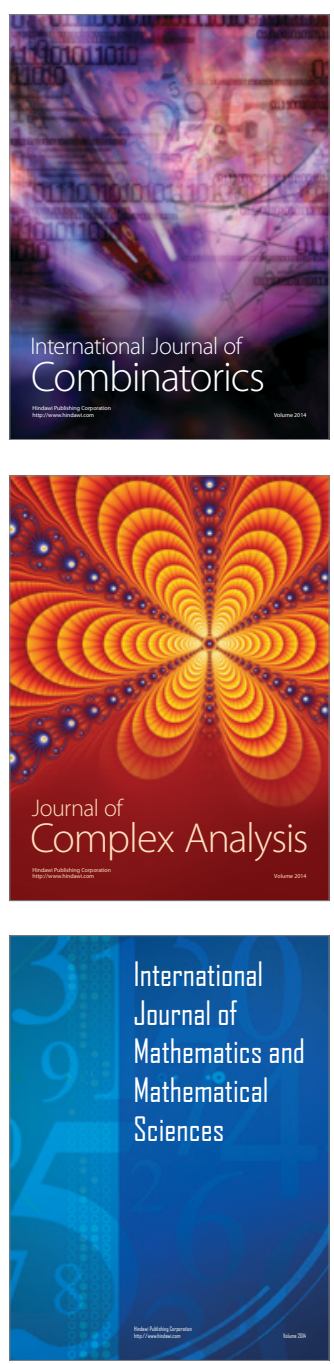
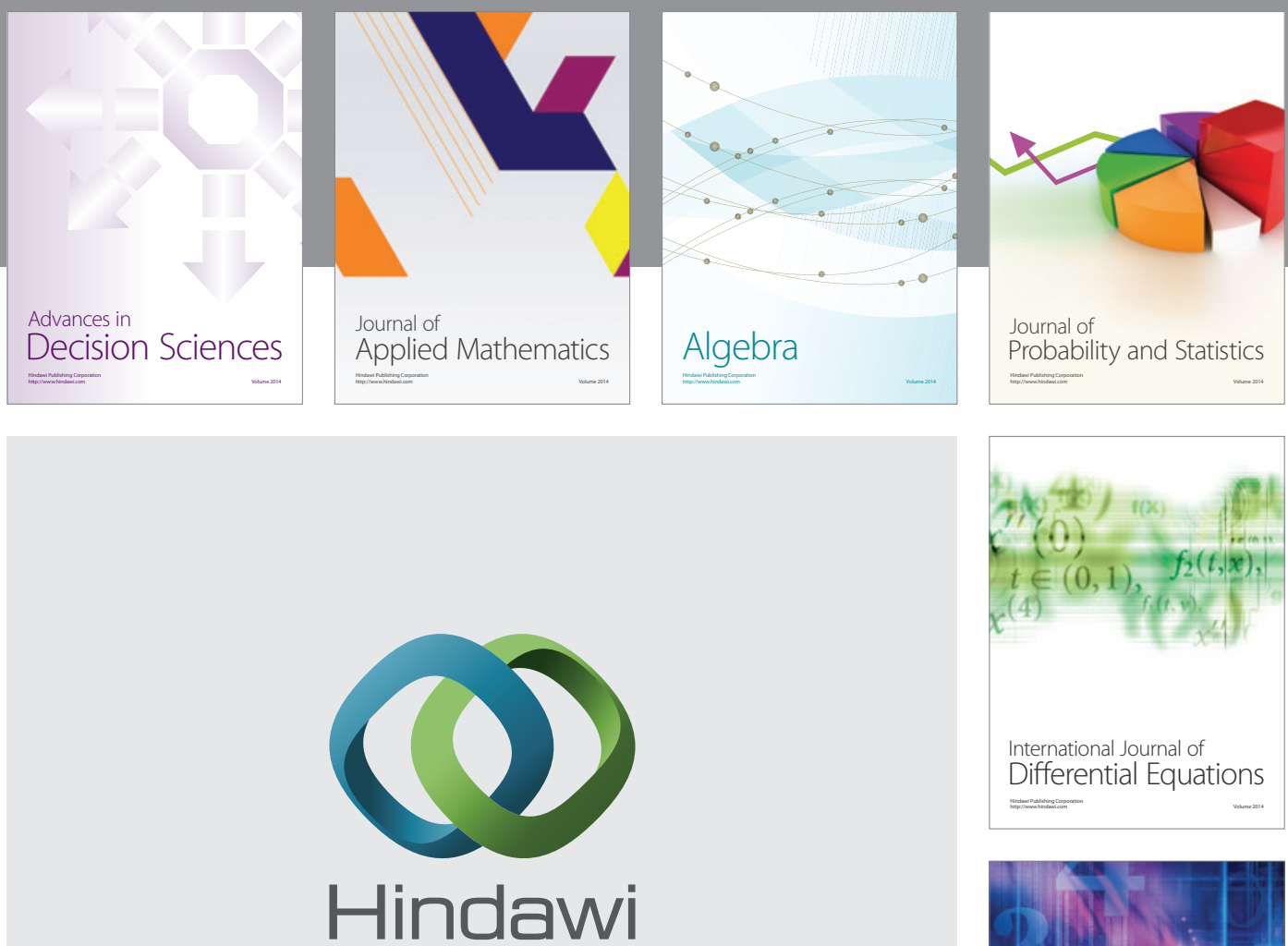

Submit your manuscripts at http://www.hindawi.com
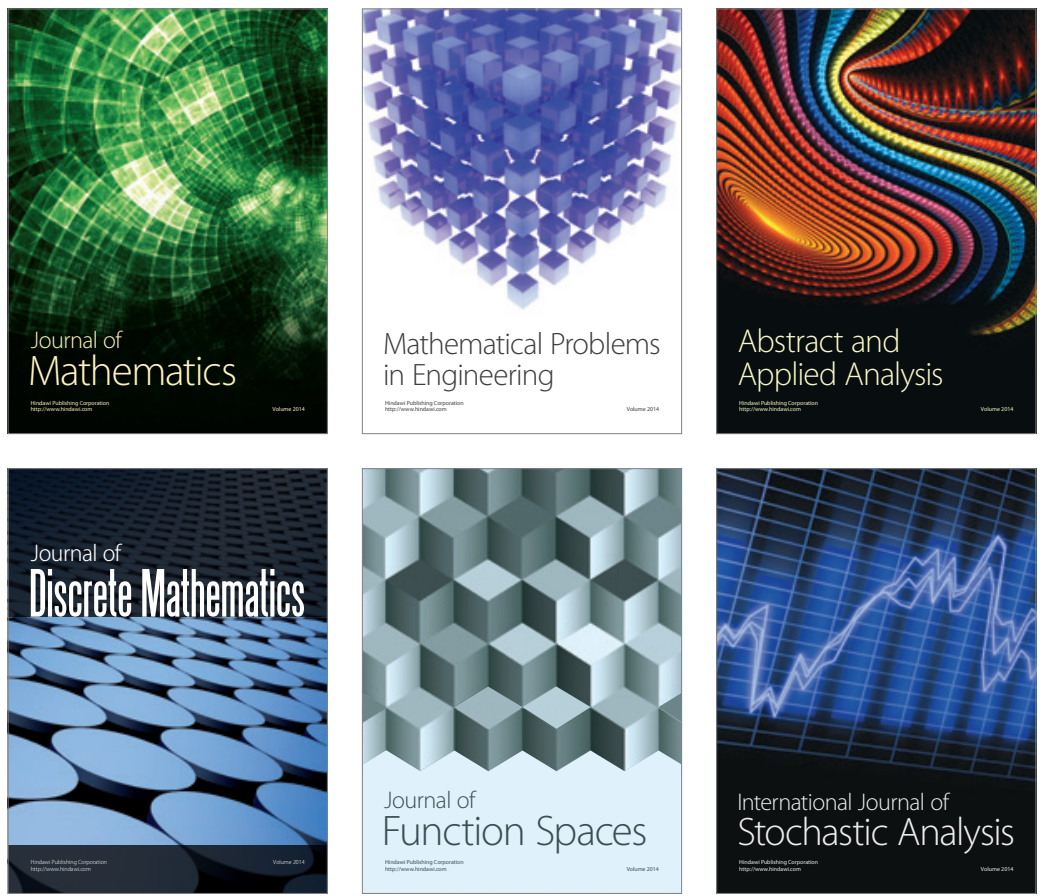

Journal of

Function Spaces

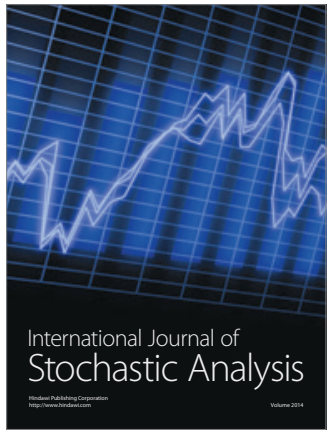

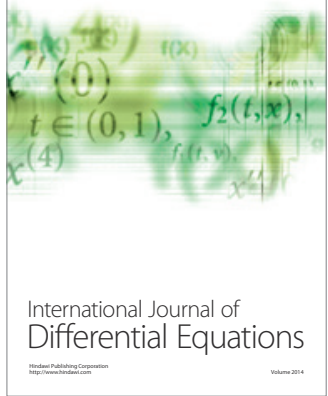
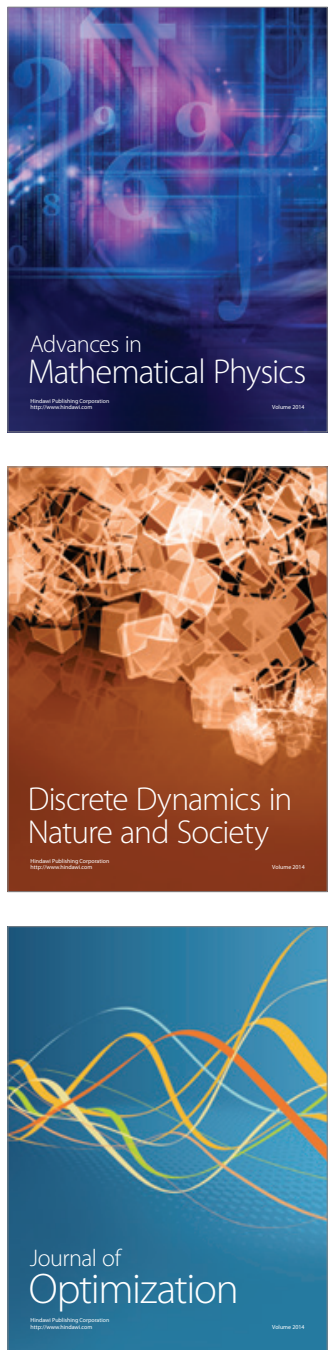